\title{
Design and Fabrication of a Novel Wideband DNG Metamaterial with the Absorber Application in Microwave X-Band
}

\author{
Ahmed Mahmood, ${ }^{1,2}$ Gölge Ögücü Yetkin, ${ }^{2}$ and Cumali Sabah ${ }^{3,4}$ \\ ${ }^{1}$ Department of Electrical Engineering, Salahaddin University, Erbil, Iraq \\ ${ }^{2}$ Department of Electrical and Electronics Engineering, University of Gaziantep, 27310 Gaziantep, Turkey \\ ${ }^{3}$ Department of Electrical and Electronics Engineering, Middle East Technical University-Northern Cyprus Campus (METU-NCC), \\ Kalkanli, 99738 Guzelyurt, TRNC, Mersin 10, Turkey \\ ${ }^{4}$ Kalkanli Technology Valley, Middle East Technical University-Northern Cyprus Campus (METU-NCC), Kalkanli, 99738 Guzelyurt, \\ TRNC, Mersin 10, Turkey
}

Correspondence should be addressed to Gölge Ögücü Yetkin; golge.ogucu@gmail.com

Received 22 November 2016; Accepted 1 March 2017; Published 26 March 2017

Academic Editor: Gary Wysin

Copyright ( 2017 Ahmed Mahmood et al. This is an open access article distributed under the Creative Commons Attribution License, which permits unrestricted use, distribution, and reproduction in any medium, provided the original work is properly cited.

A novel metamaterial, which exhibits a wideband double negative behavior in X-band, is proposed, designed, and investigated in this paper. The metamaterial is composed of modified S-shaped split-ring resonators (S-SRR). The periodic structure is designed and simulated using CST MWs. Next, the experiments are carried out, and it is shown that the simulation and the experimental results agree well and the designed structure has a wide bandwidth in X-band. An absorber application of this metamaterial is also provided, and the structure can be used as an absorber with absorption rate of over $80 \%$ for the polarization angles between $0^{\circ}$ and $40^{\circ}$.

\section{Introduction}

Metamaterials are based on periodic or nonperiodic artificial engineered structures that exhibit unusual characteristic behaviors, like negative permeability $\varepsilon$ and negative permittivity $\mu$, simultaneously over a common resonance frequency band $[1,2]$. From the first realization of the metamaterials by periodic wire and split-ring resonator (SRR) structures $[3,4]$, there are various modified shapes and geometries such as square, triangle, $\Lambda-, \Omega-, \mathrm{L}_{-}, \mathrm{S}-$, and P-shaped resonators [5-11] utilized in the designs in order to meet certain specifications in the applications. These geometries can be set in one-, two- or three-dimensional arrangements to control the electromagnetic wave propagation behavior through the medium.

The wideband metamaterials in X-band have been designed in $[10,11]$. S-shaped SRRs were printed on opposite side of the host material and transmitted power was provided to show the DNG characteristics of the designed configuration in [10]. The double P-shaped resonators in [11] were printed on single side unlike that in [10]. The $S$-parameters were presented; however there were no possible metamaterial applications found.

In this paper, a novel wideband metamaterial comprising a modified S-shaped SRR is proposed for X-band frequencies. The entire periodic structure is placed on one side of the substrate, which simplifies the manufacturing process and can be considered as an advantage of the designed structure. The retrieved constitutive parameters and the $S$-parameters obtained via simulations and experiments demonstrate that the proposed structure exhibits a wideband left-handed characteristic in X-band frequencies. An absorption application is selected and also studied for demonstration of the performance of the metamaterial. The structure can be used as an absorber with absorption rate of over $80 \%$ for the polarization angles between $0^{\circ}$ and $40^{\circ}$.

\section{Design and Simulation Conditions}

A unit cell of the proposed structure is composed of a modified S-shaped SRR printed on the front side of a square substrate of a side length of $16 \mathrm{~mm}$ and thickness of $1.6 \mathrm{~mm}$ as 


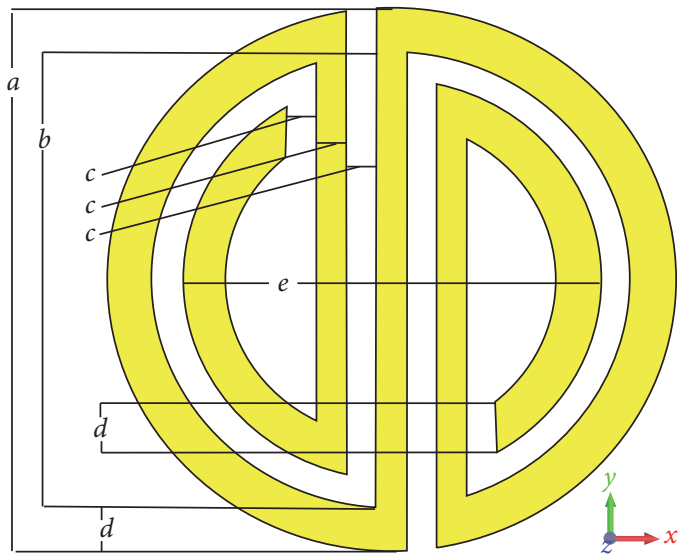

(a)

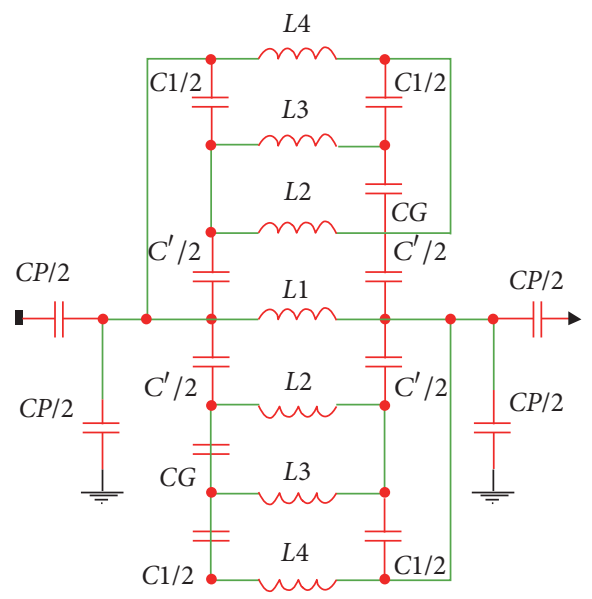

(b)

Figure 1: (a) The proposed unit cell with $a=9.69 \mathrm{~mm}, b=8.78 \mathrm{~mm}, c=0.51 \mathrm{~mm}, d=0.93 \mathrm{~mm}$, and $e=8.25 \mathrm{~mm}$. (b) Equivalent circuit model of proposed unit cell.

shown in Figure 1(a). The two ends of the S-shaped SRR are elongated in such a way that they take the form of C-shaped SRRs. In the design, FR-4 Epoxy of permittivity $\varepsilon_{r}=4.3$ and loss tangent $\delta=0.025$ is chosen as the substrate, and the copper of electrical conductivity $\sigma=5.8 \times 10^{7} \mathrm{~S} / \mathrm{m}$ with coating thickness of $0.017 \mathrm{~mm}$ is chosen for printing the SRRs. The simulations are carried out by using the commercial program CST Microwave Studio 2016. Figure 1(b) shows the $L C$ parameters that represent the electric topology of a modified S-SRR unit cell with the $\left(L, L_{1}, L_{2}, L_{3}\right)$ inductors and $\left(C^{\prime}, C_{1}, C_{p}, C_{G}\right)$ capacitors. Explicit expressions for $L$ and $C$ can be found in [12-14].

A unit cell of the proposed structure together with the ports applied in the simulations is shown in Figure 2. In the simulations, the unit cell is placed between two waveguide ports, which are perpendicular to the direction of the wave propagation, which is along negative $z$-direction. To imitate the infinite structure, perfect electric boundary (PEB) conditions are set at the boundary surfaces perpendicular to the E-field while perfect magnetic boundary (PMB) conditions are at the boundary surfaces perpendicular to the $\mathrm{H}$-field (see Figure 2). The frequency band is chosen as the $\mathrm{X}$-band region (8-12 GHz).

\section{Electric Field, Magnetic Field, and Surface Current Distributions}

Basically, the nonhomogenous metamaterial structures provide a resonant RLC circuit behavior in some certain frequency bands. For the particular geometry in this paper, the modified S-shaped SRRs behave like inductors and the gaps as capacitors. To illustrate how the structure works when placed in an electromagnetic field region, the surface current distribution is presented in Figure 3 and the magnetic and electric field densities in Figure 4 at the operation frequency of $10.75 \mathrm{GHz}$, at which the structure behaves like a left-handed

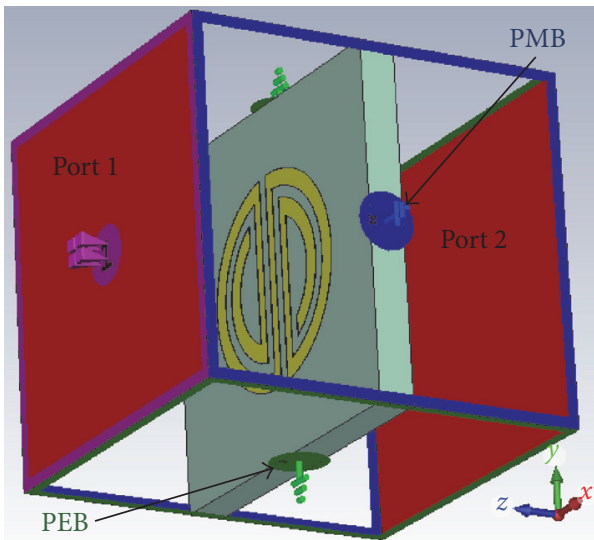

FIGURE 2: The boundary conditions of the proposed unit cell used in the simulations.

material. It can be observed from Figure 3 that the current is highly concentrated around the central wire and the copper shape structure. The magnetic field shows similar distribution as the current as shown in Figure 4(a), which supports that the resonance obtained is of magnetic type and the modified S-shaped elements are strongly coupled to this magnetic resonance. Moreover, a close examination of the current distribution in Figure 3 leads to the observation of some virtual gaps in addition to the actual gaps. These virtual gaps occur at current minimum points and this provides an additional capacitance-like response by being the voltage maxima points at the same time. The electric field is highly localized around these actual and virtual gaps as seen in Figure 4(b). The reason of high concentration around the actual gaps is the capacitive effect at these regions $[13,14]$. 


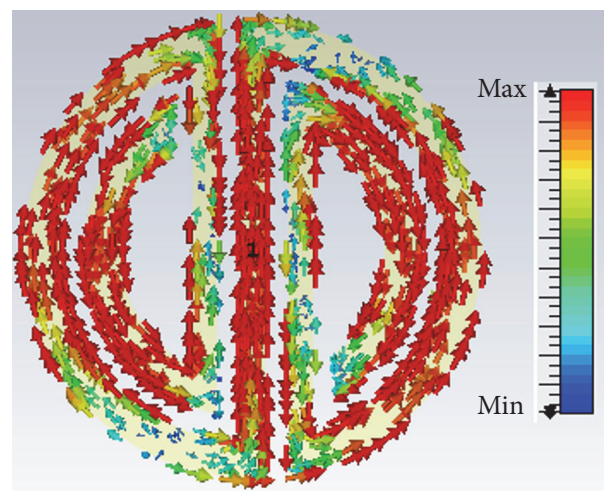

FIGURE 3: The surface current distribution at $10.75 \mathrm{GHz}$.

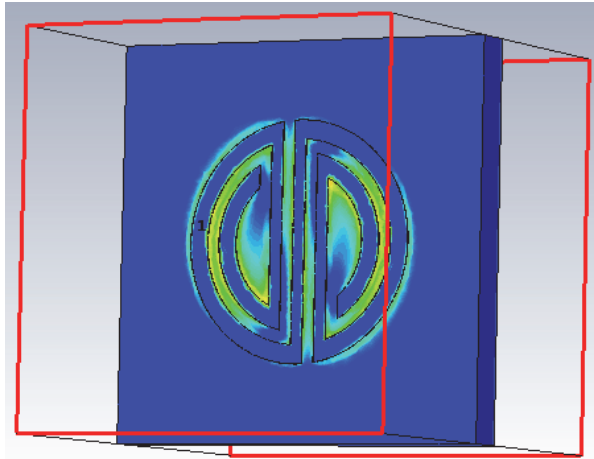

(a)

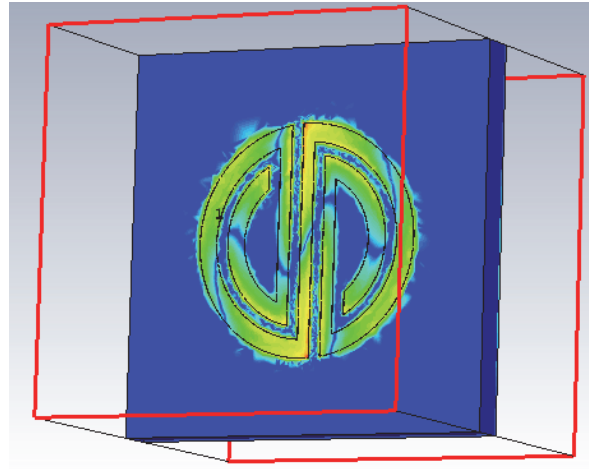

(b)

Figure 4: (a) Magnetic field and (b) electric field distributions for the proposed unit cell at $10.75 \mathrm{GHz}$.

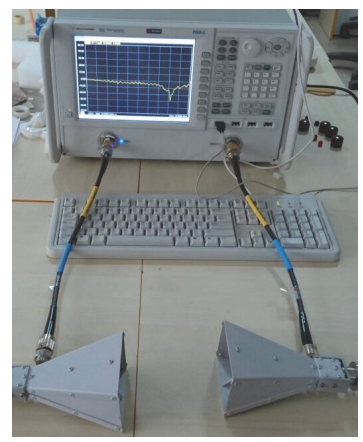

(a)

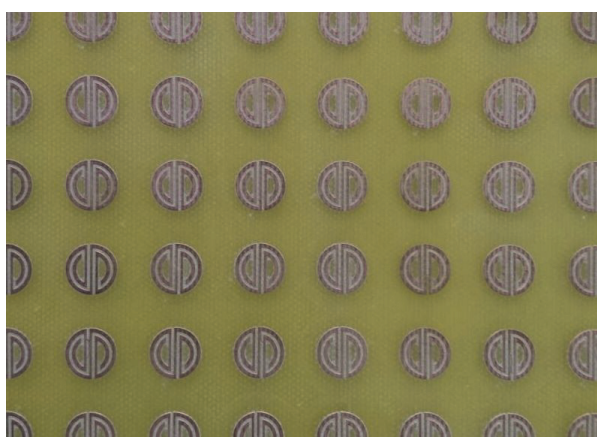

(b)

FIgURE 5: (a) Vector network analyzer, two horn antennas with coaxial cables. (b) Manufactured sample.

\section{Simulated and Experimental Results}

Before presenting the comparison of the simulated and the experimental results, it would be better to give some information about the experimental set-up. Experiment layout is prepared by placing the designed sample between two horn antennas, which are connected to a vector network analyzer (VNA) through coaxial cables as in Figure 5(a). By this way, the surface of the sample, which is shown in Figure 5(b), is guaranteed to be perpendicular to the direction of wave propagation. The metamaterial prototype is manufactured at in-house facilities using a circuit plotter with standard milling tool sets.

The incident electromagnetic wave propagates along negative $z$-axis to excite the combined materials of the sample. The electric field vector and magnetic field vector are in the $y$-axis and negative $x$-axis, respectively (see Figure 2). For calibration purposes, a measurement is conducted without the sample. After that, the sample is placed between the two horn antennas and the $S$-parameters are measured via VNA.

The simulated and the measured $S$-parameter results, which are between 8 and $12 \mathrm{GHz}$, are presented in Figure 6 . 


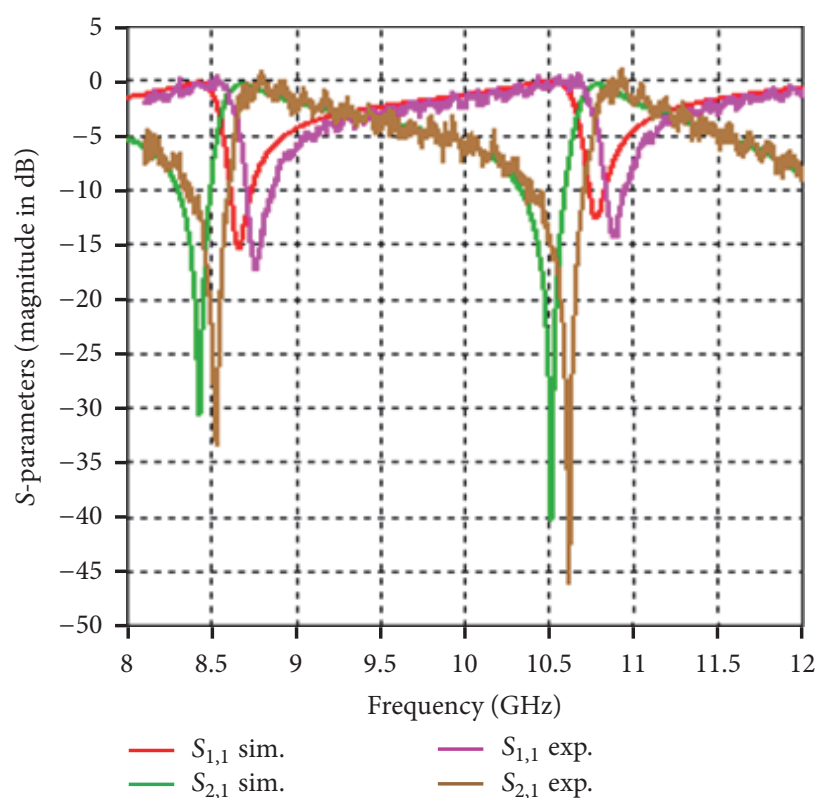

(a)

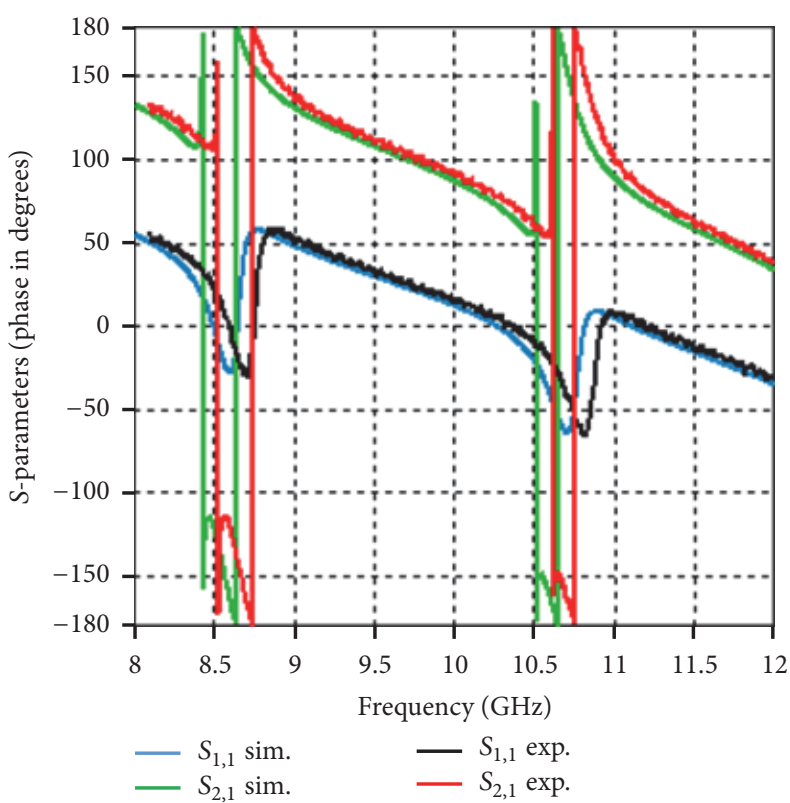

(b)

FiguRE 6: Simulated and measured $S$-parameters: (a) the magnitude (dB) and (b) the phase (degrees).

There are some small differences between the two types of obtained data, which may be due to the manufacture tolerances related to the dielectric dispersion and the etching process.

The effective permittivity and permeability values are obtained from the $S$-parameters given in Figure 6 by the use of the Nicolson-Ross-Weir (NRW) retrieval method [6, 15-17]. Using this method, the effective permittivity and permeability can be written as $\varepsilon=\left(k / k_{0}\right)((1-\Gamma) /(1+\Gamma))$ and $\mu=\left(k / k_{0}\right)((1+\Gamma) /(1-\Gamma))$, respectively, where $k_{0}$ is the propagation constant of free space. Here, $\Gamma=\left[\left(S_{11}^{2}+S_{21}^{2}+\right.\right.$ 1) $\left./ 2 S_{11}^{2}\right] \pm \sqrt{\left[\left(S_{11}^{2}+S_{21}^{2}+1\right) / 2 S_{11}^{2}\right]^{2}-1}$, and the sign is chosen such that $|\Gamma|<1$. Furthermore, $k=[\log (1 /|T|)]+j(2 m \pi-$ phase $(T)) / d$, where $T=\left[S_{11}+S_{21}-\Gamma\right] /\left[1-\Gamma\left(S_{11}+S_{21}\right)\right]$, $m=0, \pm 1, \pm 2, \pm 3, \ldots$, and $d$ is the thickness of the slab. It clarifies that the integer $m$ has multiple choices, and it is not straightforward to assign the exact solution for the permittivity and permeability. To find the exact value of the integer $m$, the group delay is calculated at each frequency, and then by taking the difference in phase of the $S_{21}$ data at adjacent frequency points and comparing it with the group delay caused with the assumed value of $m$, the convergence on the correct value of $m$ is achieved. Thus, NRW method can be applied with justification.

Figure 7 presents the simulated and the experimental results for the real parts of the effective permittivity and permeability together with the real part of the refractive index of the structure. As can be observed, the results compare well, and the structure has negative real parts in the frequency bands $\sim 8-10.45 \mathrm{GHz}$ and $\sim 10.65-11.75 \mathrm{GHz}$ for the permittivity and in the frequency band $\sim 8.5-12 \mathrm{GHz}$ for the permeability. Thus the proposed structure exhibits a wideband metamaterial behavior.

\section{Absorber Application}

To investigate the performance of the proposed S-shaped SRR, the absorption characteristics of the structure are studied both experimentally and through simulations. The absorption of a material is function of frequency and can be formulated by $A(\omega)=1-R(\omega)-T(\omega)$, where $R(\omega)=\left|S_{11}\right|^{2}$ and $T(\omega)=\left|S_{21}\right|^{2}$ represent the reflection and the transmission characteristics, respectively [18]. In order to maximize the absorption, both the reflection and the transmission can be minimized at the resonance frequency. In this study, a copper layer on the backside of the substrate material is added, so that the absorption expression is reduced to $A(\omega) \approx$ $1-R(\omega)$, and it almost depends only on reflection now [1820].

Figure 8(a) shows the VNA and the experiment alignment setting. After the calibration test without the testing sample, horn antenna is used to illuminate the sample in the $\mathrm{X}$ band frequency regime, which is connected with VNA by a coaxial cable. As can be seen in Figure 8(b), the measurement results show good agreement with the simulation results. A maximum in the absorption ratio is about $99.99 \%$ at $8.94 \mathrm{GHz}$ experimentally. Both the experimental and the simulation results suggest that the proposed structure can be used for perfect absorption applications. Note that the discrepancies between the experimental and simulation data are imputed to fabrication tolerances and dielectric dispersion of the substrate. The misalignment during the experiment may also be considered as another source of error. 


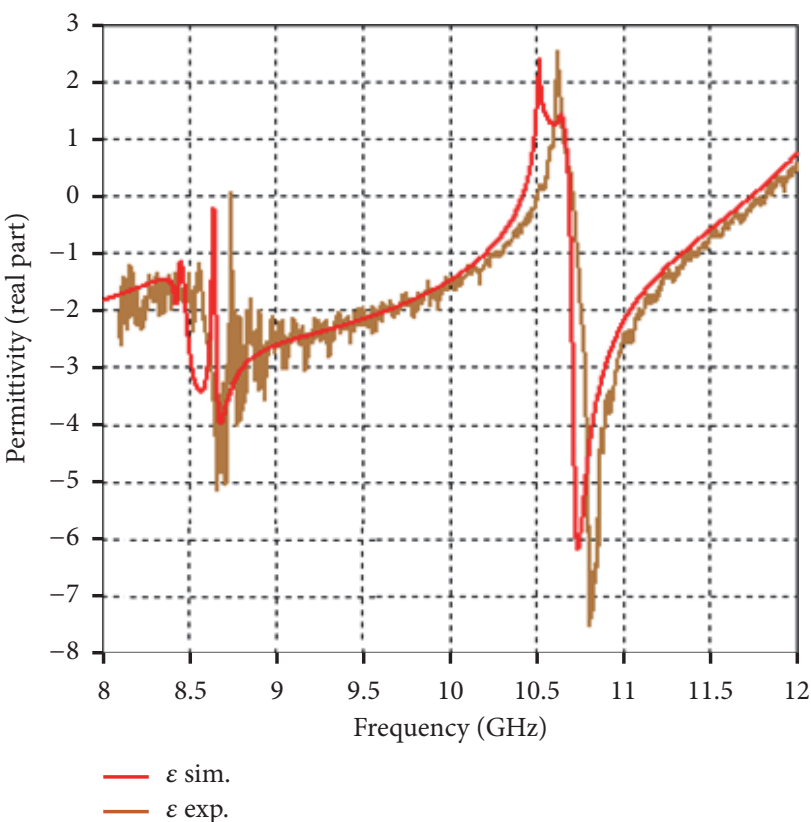

(a)

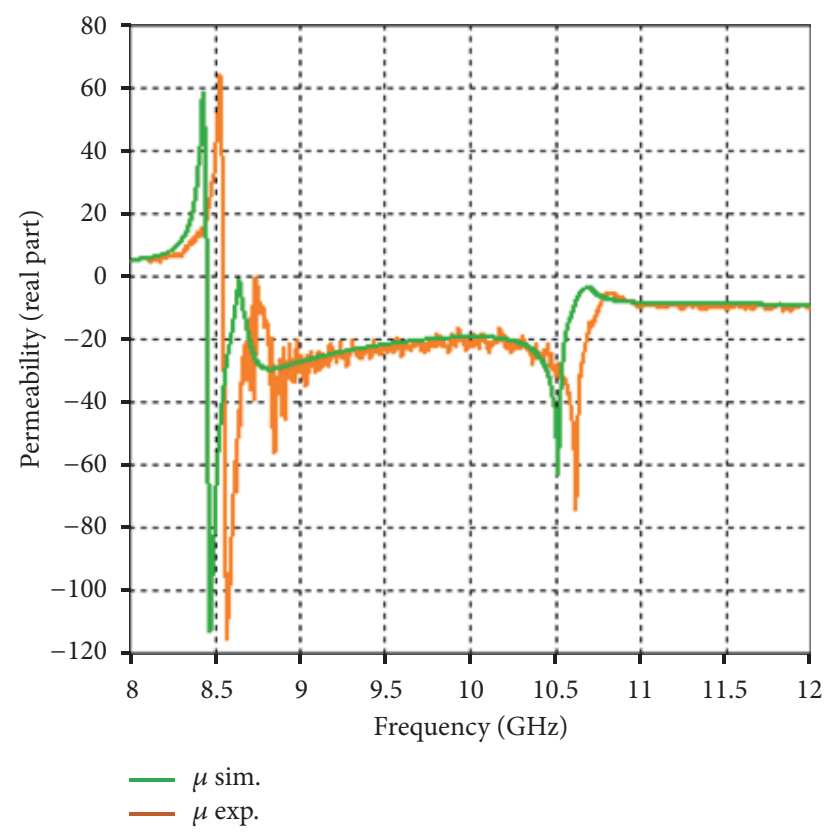

(b)

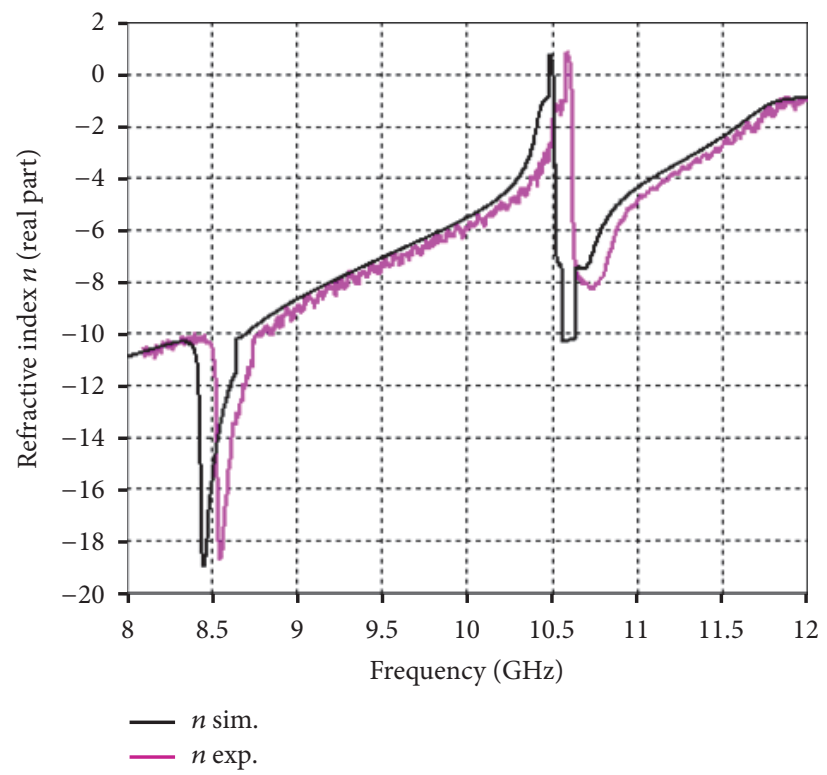

(c)

FIGURE 7: The simulated and experimental results for the real parts of (a) permittivity, (b) permeability, and (c) refractive index.

Furthermore, the $S$-parameter and the absorption characteristics are simulated for different polarization angles in 8.75-9.05 GHz frequency band. In Figure 9, $S_{11}$ is presented for normal and oblique incidences. It is observed that reflection achieves its lowest value in normal incidence case and the resonance occurs around $8.912 \mathrm{GHz}$. As the polarization varies from normal to oblique incidence, the reflection also varies and a stable frequency response can be obtained for $\theta<40^{\circ}$ when $\phi=0^{\circ}$.
In Figure 10, the absorption characteristics are provided in the frequency band of $8.75-9.05 \mathrm{GHz}$ for the polarization angle $\theta$ between $0^{\circ}$ and $40^{\circ}$. It can be deduced that the proposed metamaterial can be used as a narrow band, $175 \mathrm{MHz}$ perfect absorber with absorption over $90 \%$ in $8.825-9.0 \mathrm{GHz}$. At the resonance frequency around $8.912 \mathrm{GHz}, S_{11}$ is 0.0039 . Correspondingly, the absorption rate is $99.99 \%$ at the resonance and the device can be used as a perfect absorber at the mentioned frequency. 


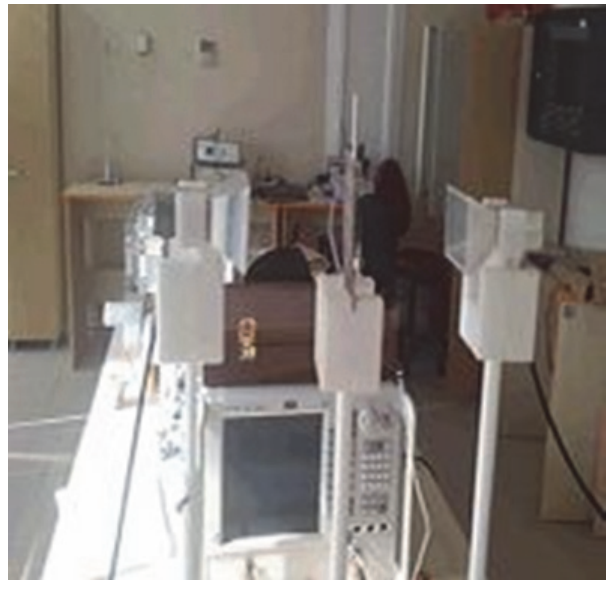

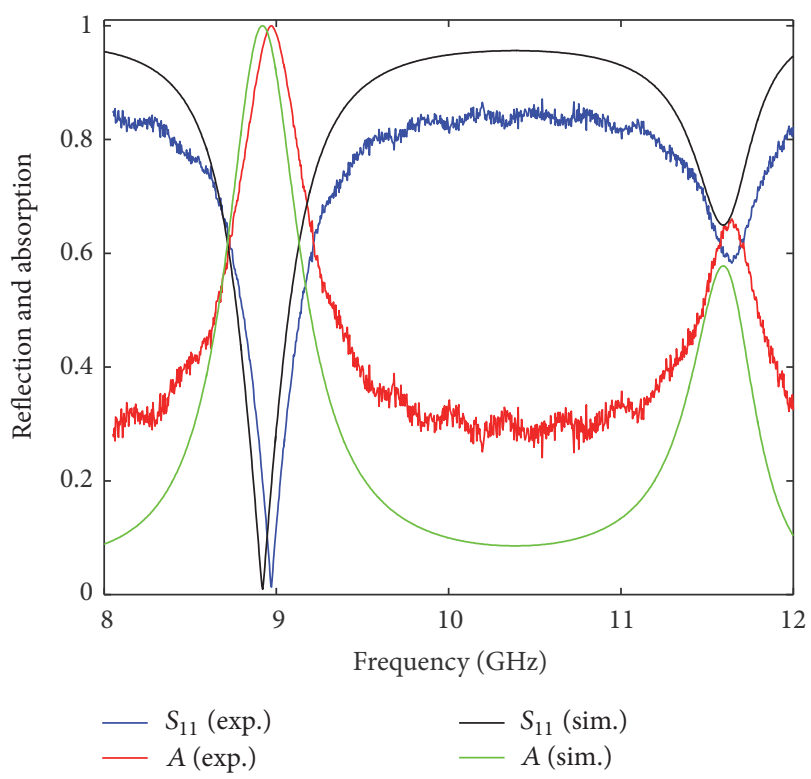

(b)

FiguRE 8: (a) Absorption experiment setting. (b) Simulated and measured results of reflection and absorption ratio.

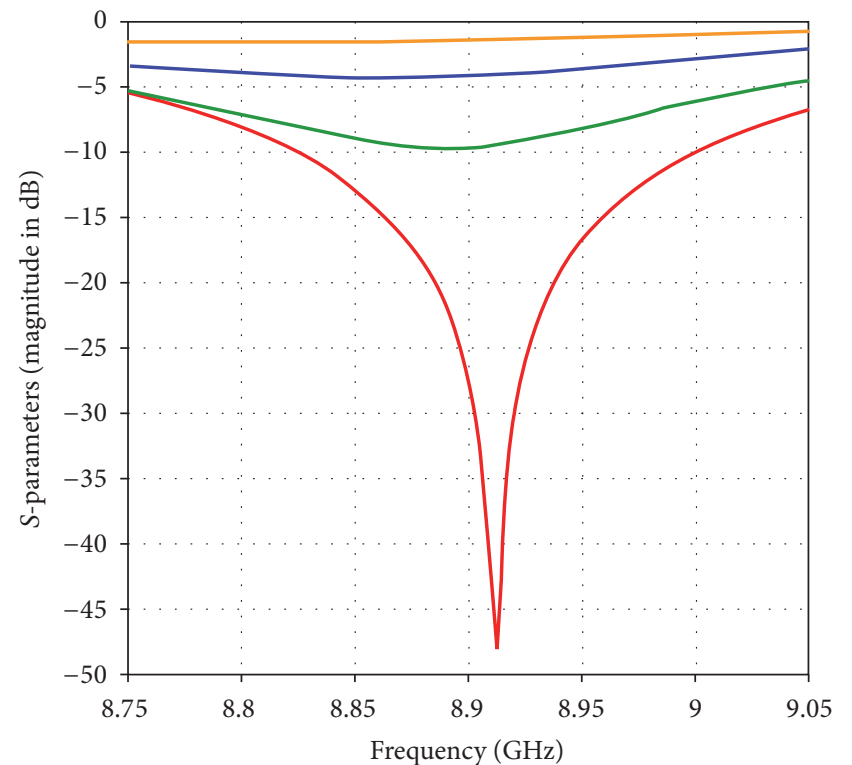

$-\phi=0, \theta=0$
$-\phi=10, \theta=0 \quad \longrightarrow \phi=20, \theta=0$
$\phi=30, \theta=0$

(a)

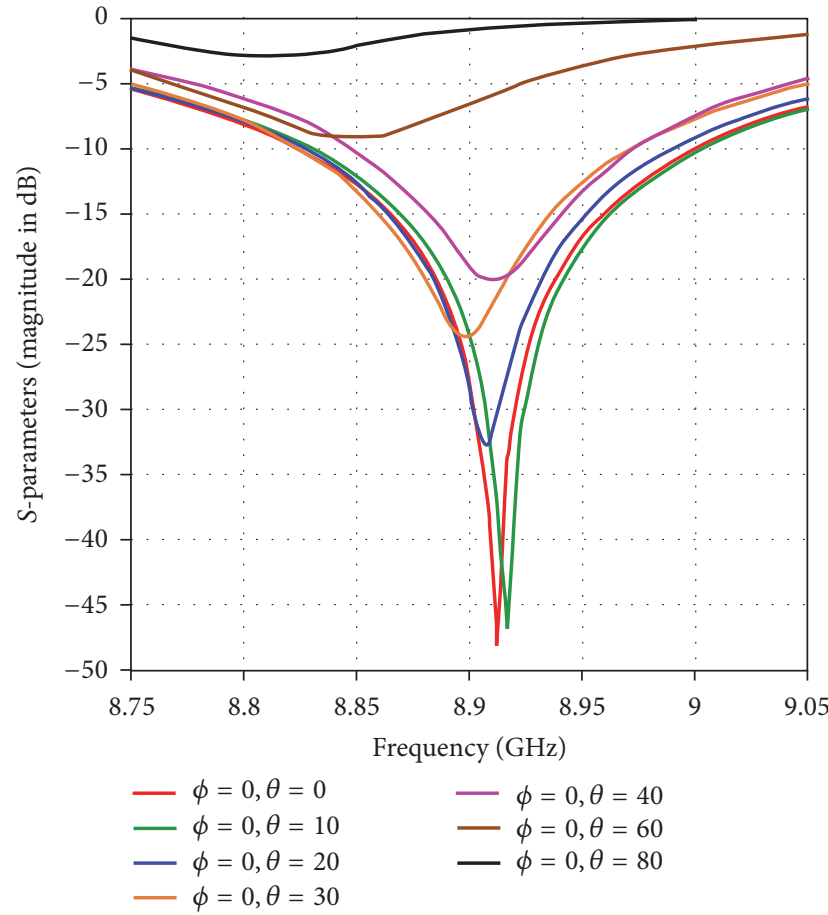

(b)

FIGURE 9: Frequency response of $S_{11}$ for different polarization angles: (a) $\theta=0^{\circ}$ and $\phi$ varies and (b) $\theta$ varies and $\phi=0^{\circ}$.

\section{Conclusion}

In this work, a modified S-shape ring resonator in periodic arrangement is introduced and investigated both numerically and experimentally. Based on the experiment results obtained, it has been demonstrated that the proposed structure exhibits wideband DNG characteristics in the frequency band of interest. In other words, the experimented novel metamaterial is well designed and successfully operates around the resonance frequency to provide simultaneously 


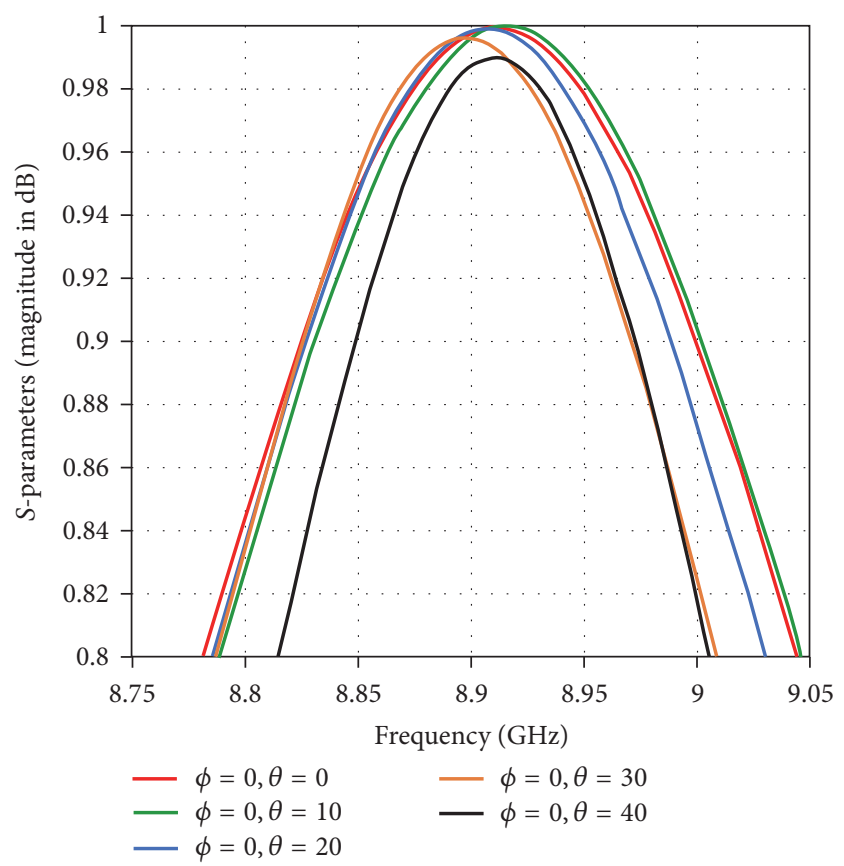

(a)

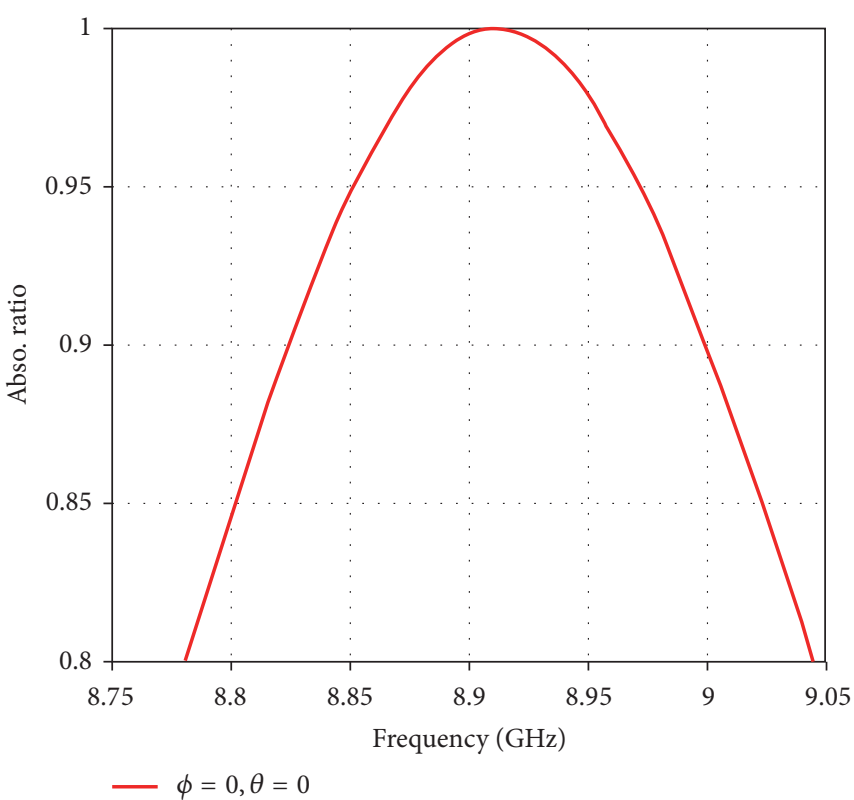

(b)

FIgURE 10: Absorption for different polarization angles: (a) $\theta$ varies and $\phi=0^{\circ}$ and (b) $\theta=0^{\circ}$ and $\phi=0^{\circ}$.

double negative permittivity and permeability in X-band. Additionally, simple fabrication of this novel metamaterial is another design advantage.

An absorber application has also been studied and measured. The measurement results show good agreement with the simulation results. Additionally, the simulated results for $S$-parameters and the absorption rate are obtained at different polarization angles. It has been shown that high absorption can be achieved for oblique incidences up to $40^{\circ}$ and with absorption peak more than $90 \%$ between 8.825 and $9 \mathrm{GHz}$. A perfect absorber can be obtained at the resonance frequency around $8.912 \mathrm{GHz}$.

\section{Conflicts of Interest}

The authors declare that there are no conflicts of interest regarding the publication of this paper.

\section{References}

[1] V. G. Veselago, "The electrodynamics of substances with simultaneously negative values of $\varepsilon$ and $\mu$," Soviet Physics Uspekhi, vol. 10, no. 4, pp. 509-514, 1968 .

[2] J. B. Pendry, A. J. Holden, W. J. Stewart, and I. Youngs, "Extremely low frequency plasmons in metallic mesostructures," Physical Review Letters, vol. 76, no. 25, pp. 4773-4776, 1996.

[3] D. R. Smith, W. J. Padilla, D. C. Vier, S. C. Nemat-Nasser, and S. Schultz, "Composite medium with simultaneously negative permeability and permittivity," Physical Review Letters, vol. 84, no. 18 , pp. 4184-4187, 2000.
[4] R. A. Shelby, D. R. Smith, and S. Schultz, "Experimental verification of a negative index of refraction," Science, vol. 292, no. 5514, pp. 77-79, 2001.

[5] P. Markoš and C. M. Soukoulis, "Numerical studies of lefthanded materials and arrays of split ring resonators," Physical Review E, vol. 65, no. 3, Article ID 036622, 2002.

[6] C. Sabah, "Electric and magnetic excitations in anisotropic broadside-coupled triangular-split-ring resonators," Applied Physics A: Materials Science and Processing, vol. 108, no. 2, pp. 457-463, 2012.

[7] C. Sabah and F. Urbani, "Experimental analysis of $\Lambda$-shaped magnetic resonator for mu-negative metamaterials," Optics Communications, vol. 294, pp. 409-413, 2013.

[8] J. H. Lv, X. W. Hu, M. H. Liu, B. R. Yan, and L. H. Kong, "Negative refraction of a double L-shaped metamaterial," Journal of Optics A: Pure and Applied Optics, vol. 11, no. 8, Article ID 085101, 2009.

[9] J. Huangfu, L. Ran, H. Chen et al., "Experimental confirmation of negative refractive index of a metamaterial composed of $\Omega$ like metallic patterns," Applied Physics Letters, vol. 84, no. 9, pp. 1537-1539, 2004.

[10] H. Chen, L. Ran, J. Huangfu et al., "Left-handed materials composed of only S-shaped resonators," Physical Review E, vol. 70, no. 5, Article ID 57605, 2004.

[11] D.-L. Jin, J.-S. Hong, and H. Xiong, "A novel single-sided wideband metamaterial," Applied Computational Electromagnetics Society Journal, vol. 27, no. 12, pp. 971-976, 2012.

[12] J. Naqui, J. Coromina, A. Karami-Horestani, C. Fumeaux, and F. Martín, "Angular displacement and velocity sensors based on coplanar waveguides (CPWs) loaded with S-shaped split ring resonators (S-SRR)," Sensors, vol. 15, no. 5, pp. 9628-9650, 2015. 
[13] H. S. Chen, L. X. Ran, J. T. Huangfu et al., "Magnetic properties of S-shaped split-ring resonators," Progress in Electromagnetics Research, vol. 51, pp. 231-247, 2005.

[14] A. K. Horestani, M. Duran-Sindreu, J. Naqui, C. Fumeaux, and F. Martin, "Coplanar waveguides loaded with s-shaped split-ring resonators: modeling and application to compact microwave filters," IEEE Antennas and Wireless Propagation Letters, vol. 13, pp. 1349-1352, 2014.

[15] A. M. Nicolson and G. F. Ross, "Measurement of the intrinsic properties of materials by time-domain techniques," IEEE Transactions on Instrumentation and Measurement, vol. 19, no. 4, pp. 377-382, 1970.

[16] W. B. Weir, "Automatic measurement of complex dielectric constant and permeability at microwave frequencies," Proceedings of the IEEE, vol. 62, no. 1, pp. 33-36, 1974.

[17] V. Milosevic, B. Jokanovic, and R. Bojanic, "Effective electromagnetic parameters of metamaterial transmission line loaded with asymmetric unit cells," IEEE Transactions on Microwave Theory and Techniques, vol. 61, no. 8, pp. 2761-2772, 2013.

[18] N. I. Landy, S. Sajuyigbe, J. J. Mock, D. R. Smith, and W. J. Padilla, "Perfect metamaterial absorber," Physical Review Letters, vol. 100, no. 20, Article ID 207402, 2008.

[19] F. Dinçer, M. Karaaslan, and C. Sabah, "Design and analysis of perfect metamaterial absorber in $\mathrm{GHz}$ and $\mathrm{THz}$ frequencies," Journal of Electromagnetic Waves and Applications, vol. 29, no. 18, pp. 2492-2500, 2015.

[20] F. Dincer, O. Akgol, M. Karaaslan, E. Unal, and C. Sabah, "Polarization angle independent perfect metamaterial absorbers for solar cell applications in the microwave, infrared, and visible regime," Progress in Electromagnetics Research, vol. 144, pp. 93101, 2014. 

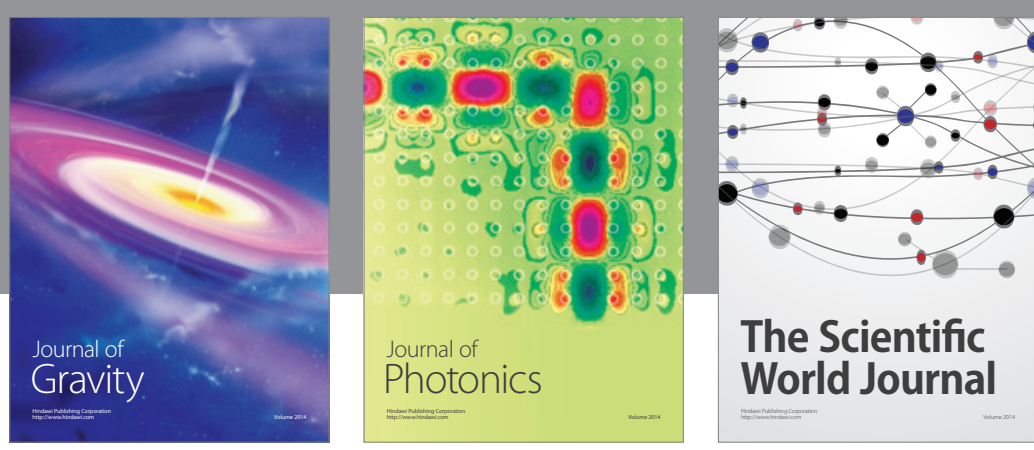

The Scientific World Journal
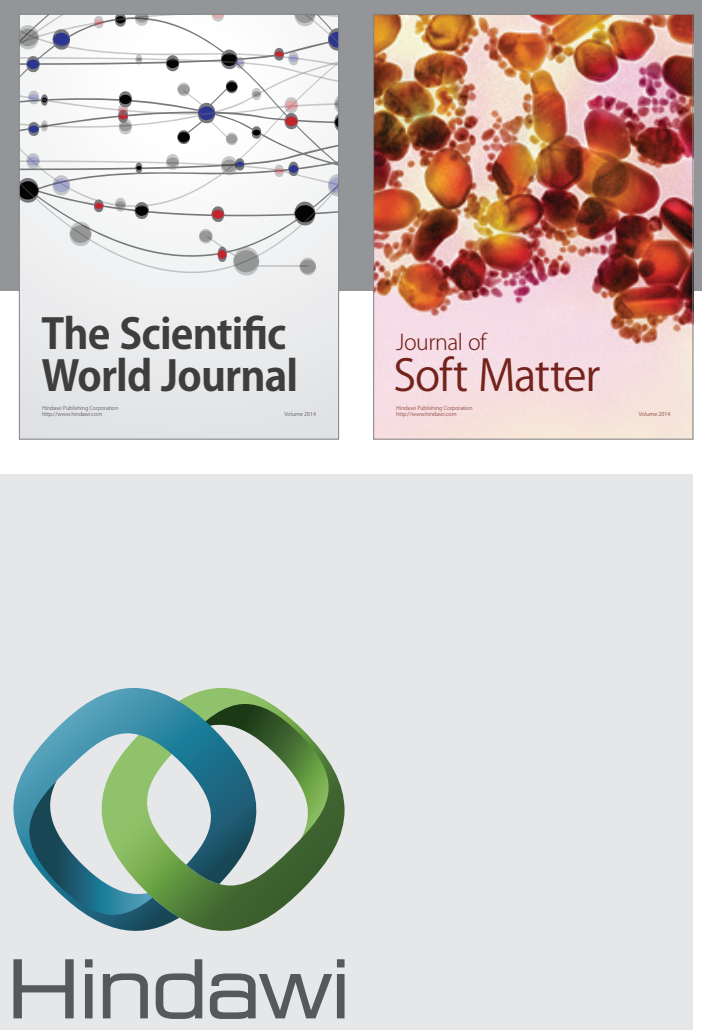

Submit your manuscripts at

https://www.hindawi.com
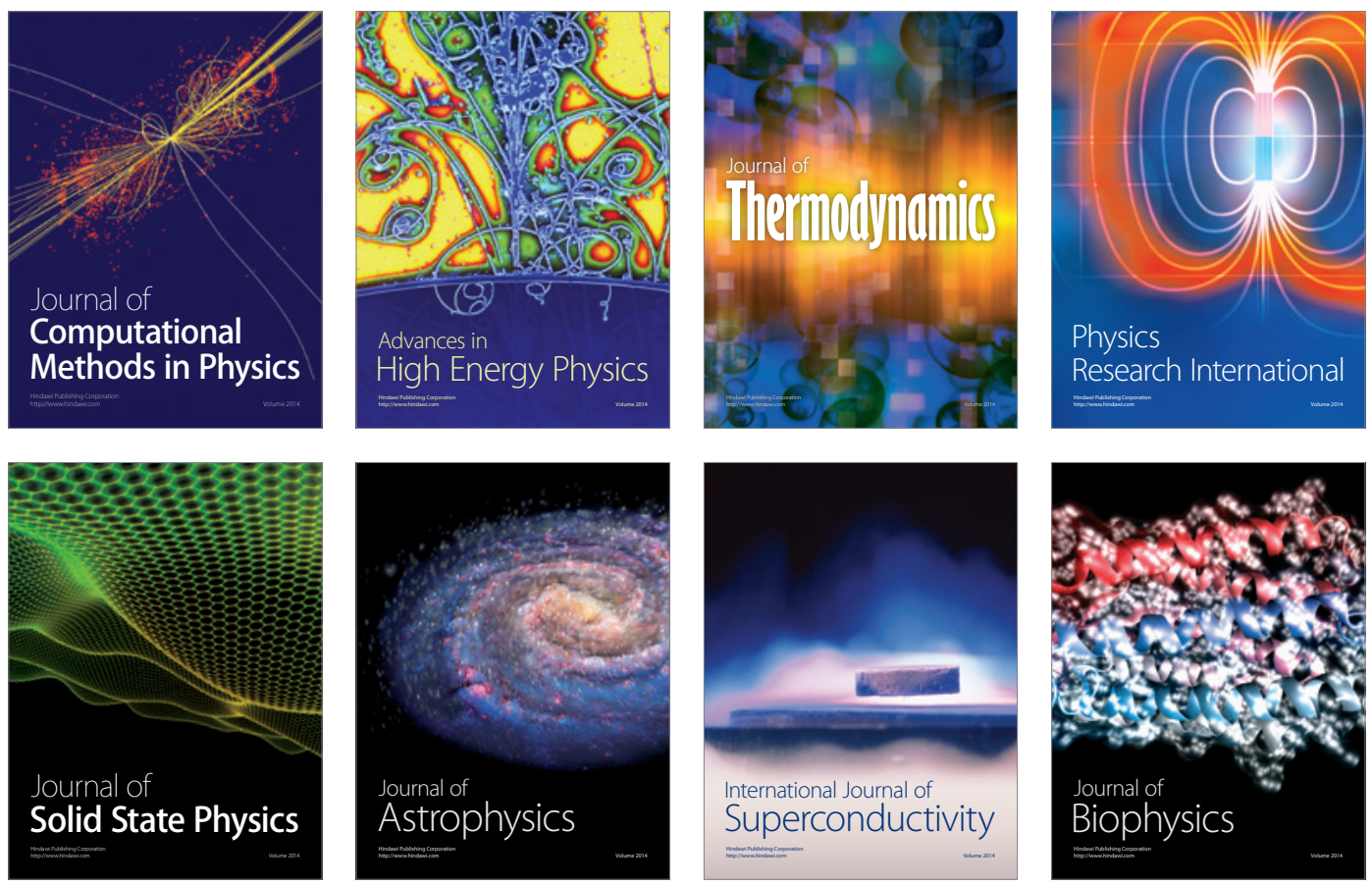
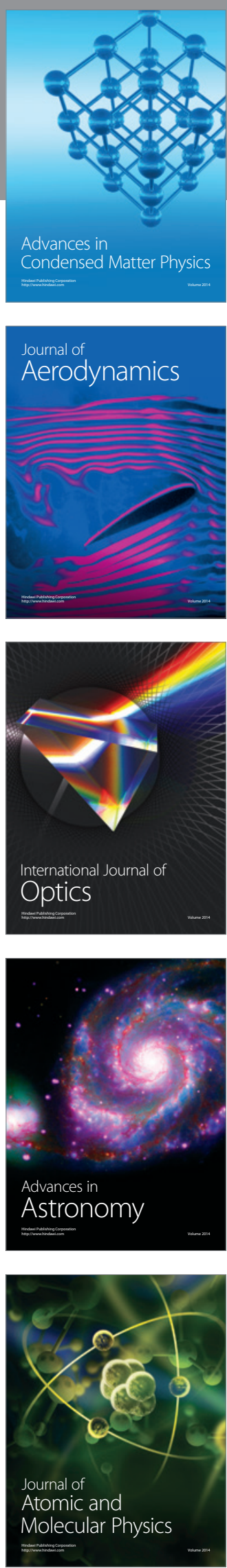